\title{
Higher Prevalence of Prostate Cancer among Veterans with Type 2 Diabetes
}

\author{
Subhashini Yaturu*, Nagwa Benyamin, Barbara Youngberg, Michael Marchese \\ Dorn VA Medical Center/USC School of Medicine, Columbia, SC, USA \\ Email: *yaturu@yahoo.com
}

How to cite this paper: Yaturu, S., Benyamin, N., Youngberg, B. and Marchese, M. (2017) Higher Prevalence of Prostate Cancer among Veterans with Type 2 Diabetes. Journal of Diabetes Mellitus, 7, 316-320.

https://doi.org/10.4236/jdm.2017.74026

Received: September 18, 2017

Accepted: November 13, 2017

Published: November 16, 2017

Copyright () 2017 by authors and Scientific Research Publishing Inc. This work is licensed under the Creative Commons Attribution International License (CC BY 4.0).

http://creativecommons.org/licenses/by/4.0/

\begin{abstract}
Background: Diabetes mellitus and cancer are common conditions. Based on epidemiological evidence, American Diabetes Association (ADA) and the American Cancer Society (ACS) concluded that patients with diabetes are at a higher risk for cancers in their joint position statement. That statement includes a meaningful association between cancer and diabetes; common risk factors for both; possible biological links between the two and also whether the diabetes therapy alters risk or prognosis of cancer. Additional comments included lack of association or evidence between diabetes and cancers such as lung cancer and lymphomas; and reduced risk of prostate cancer among subjects with type 2 diabetes. It was also stated that some cancers such as liver, pancreas, colon and rectum, breast, bladder and endometrial cancers. Since prevalence of diabetes is more than 25\% among Veterans, our aim of this study was to evaluate the prevalence of various cancers among Veterans with type 2 diabetes at Albany, NY. Methods: In a single center, retrospective chart review study was carried out using computerized patient records, after the Institutional Review Board and Research and development review board at Veterans health administration at Stratton VA MC at Albany, New York. Using CPT codes and verification by review of clinical and laboratory information, the study was carried out. The information collected include the BMI, smoking status, duration of diabetes, medications used, other medical problems such as hypertension, atherosclerotic cardiovascular disease, various cancers; laboratory data included $\mathrm{HbAlC}$, eGFR, Creatinine and lipid parameters. Results: The study identified 6777 subjects with type 2 diabetes with a mean age of $61 \pm 10$ and BMI of $32.6 \pm 6$. Total of 1046 subjects identified with cancers, after exclusion of skin cancers. The most common cancers among Veterans with type $2 \mathrm{DM}$ are prostate cancer, colon cancer, bladder cancer and Lung Cancer. Other cancers include renal cell cancer, liver cancer, pancreatic cancer, lymphomas and breast cancer. Conclusion: It is worth considering the causes why prostate and bladder cancers are so common among subjects
\end{abstract}


with diabetes using national database.

\section{Keywords}

Diabetes, Cancer, Prostate Cancer, Colon Cancer, Kung Cancer

\section{Introduction or Background}

Diabetes and cancer are common conditions with increase in prevalence globally. Prevalence of diabetes among Veterans is more than $25 \%$ compared to $8 \%$ 9\% globally. Epidemiological data links diabetes to increased risk for cancers such as colorectal, pancreatic cancer, hepatoma, pancreatic cancer, bladder cancer; endometrial and breast cancer in women. American Diabetes Association (ADA) and the American Cancer Society (ACS), based on epidemiological evidence, concluded that patients with diabetes are at a higher risk for cancers in their joint position statement [1] [2]. The statement included whether there is a meaningful association between cancer and diabetes; common risk factors for both; possible biological links between the two and whether the diabetes therapy alters risk or prognosis of cancer. Lack of association or evidence between diabetes and cancers are lung cancer and lymphomas [1]. It is considered that prostate cancer is less common in subjects with diabetes. In a meta-analysis, it is reported that there is a strong evidence of an inverse association between diabetes and prostate cancer [3]. In a population-based study from United Kingdom [4], the authors reported the association of type 2 diabetes with an increased risk of prostate cancer mortality (23\%) and all-cause mortality (25\%). In a study based on meta-analysis, pre-existing type 2 diabetes was reported to be associated with all-cause mortality [5]. More recent data indicate that diabetes did not adversely impact survival in patients with prostate cancer [6] [7] [8]. Since prevalence of diabetes is more than $25 \%$ among Veterans, our aim of this study was to evaluate the prevalence of various cancers among Veterans with type 2 diabetes at Albany, NY.

\section{Methods}

This is a single-center clinical, retrospective data base study. This clinical study part was carried out after the approval of the protocol by the Institutional Review Board as well as Research and development committee at Stratton VAMC in Albany, New York. The study population consists of all patients diagnosed with type 2 diabetes and seen at Stratton VAMC between 2010-2012 (both inclusive). The study included 6877 individuals. Most of the subjects were men $(>95 \%)$. Patients were not recruited, as it is a retrospective chart review study. Subjects were identified using diagnostic CPT codes for type 2 diabetes. No distinction on racial or ethnic basis. No controls were included as the study evaluated prevalence of various cancers in subjects with type 2 diabetes. Skin can- 
cers were not included in the list of cancer data collected. The data collected from Computerized patient record system include history of smoking, alcohol abuse, duration of diabetes, list of other medical problems including all cancers, list of medications; clinical details such as age, sex, height, weight, body mass index (BMI), blood pressure. The laboratory data collected include hemoglobin A1C. Cancer data was available either by work up at VAMC or by scanned documents into VA medical records when work up was done outside VAMC.

\section{Statistical Analysis}

Statistical analyses were carried out using Microsoft Excel 2007. All data are presented as means \pm S.D. A $p$ value of $<0.05$ is considered statistically significant. Descriptive statistics of the demographic, clinical and biochemical data was generated and tabulated. Data collected is expressed as means and standard deviation of the mean. Difference in the clinical and biochemical parameters between the subjects with associated cancer and those without any documented cancer was carried out.

\section{Results}

Baseline characteristics of the cohort are shown in Table 1. The study included 6778 subjects with type 2 diabetes. Their average age was 59 years, and most were Caucasian men. The list of cancers identified included colon cancer, lung, liver, pancreatic cancer, thyroid cancer, renal cell carcinoma, breast cancer; lymphoma and prostate cancer. The frequency of the listed cancers are listed in Table 2. The duration of diabetes at the time of diagnosis of cancer was included as the duration of diabetes. Interstingly noted prevalence of polyposis coli noted in $15 \%$ of them.

\section{Discussion}

Based on the epidemiological data, joint consensus statement American Diabetes Association and the American Cancer Society indicate that diabetes (primarily type 2) is associated with an increased risk of some cancers (liver, pancreas, en-

Table 1. Shows the Clinical details of the subjects with type 2 diabetes.

\begin{tabular}{cc}
\hline & Mean \pm SD \\
\hline Age (Years) & $59 \pm 11$ \\
BMI & $32.7 \pm 6.7$ \\
DM dur (Years) & $3.2 \pm 1.6$ \\
HTN (\%) & 88 \\
IHD (\%) & 31 \\
CKD (\%) & 16 \\
Smoker (\%) & 43
\end{tabular}


Table 2. Showing the prevalence of various cancers among all subjects with various cancers among subjects with diabetes.

\begin{tabular}{ccc}
\hline Site & Total \# & $\%$ \\
\hline Prostate & 497 & 46 \\
Colon Ca & 154 & 14 \\
Bladder & 150 & 14 \\
Lung & 131 & 12.2 \\
Kidney & 35 & 3.3 \\
Liver & 29 & 2.7 \\
Non-Hodgkins Lymphoma & 24 & 2.2 \\
Pancreas & 19 & 1.8 \\
Thyroid cancer & 19 & 1.77 \\
Breast & 18 & 1.7 \\
\hline
\end{tabular}

dometrium, colon/rectum, breast, and bladder). Diabetes is said to be associated with a reduced risk of prostate cancer [1] [2]. Data from centers for disease control (CDC) indicate that prostate cancer is the most common cancer in men and $2^{\text {nd }}$ most common cancer among men and women combined.

(https://nccd.cdc.gov/uscs/toptencancers.aspx\#text). In 2011, data base study reported that the men with diabetes were $7 \%$ less likely to develop cancer [9]. In addition, they stated that there was increased risks of cancers of the colon, rectum, liver, biliary tract, pancreas, and kidney as well as increased risks of melanoma and of leukemia [9]. The current prevalence of diabetes among Veterans is $>25 \%$ but in their study, prevalence of diabetes appears to be around $15 \%$ in their study.

In contrast to the belief that prostate cancer is less commonly association in subjects with diabetes, we noted significantly higher frequency of prostate cancer association in subjects with diabetes compared to the other cancers. The data was not geared to consider morbidity and mortality issues. In a Korean study prevalence of prostate cancer increased in relation to BMI in patients with diabetes. Diabetes may be an important predictor of the presence of high-risk prostate cancer in men with benign prostatic hypertrophy [10]. The reasons for higher frequency of prostate cancer among Veterans is not clear. Is it possible whether the risk factors that predispose Veterans for higher frequency of diabetes may be the link for prostate cancer too is a hypothesis.

\section{Conclusion}

Prostate cancer is common among veterans with diabetes.

\section{Acknowledgements}

The authors thank Ms Barbara Youngberg for excellent editing of this manuscript. None of the authors has any financial interest in publication of this ma- 
nuscript or has received any money from any other sources than the Veterans Health Administration and PRI. Dr. Yaturu receives salary support from VA.

\section{References}

[1] Giovannucci, E., et al. (2010) Diabetes and Cancer: A Consensus Report. Diabetes Care, 33, 1674-1685. https://doi.org/10.2337/dc10-0666

[2] Giovannucci, E., et al. (2010) Diabetes and Cancer: A Consensus Report. CA: $A$ Cancer Journal for Clinicians, 60, 207-221. https://doi.org/10.3322/caac.20078

[3] Weir, A.N., et al. (1989) A High-Performance Liquid Chromatography Method for the Simultaneous Assay of Diaminopimelate Epimerase and Decarboxylase. Analytical Biochemistry, 180, 298-302. https://doi.org/10.1016/0003-2697(89)90434-X

[4] Bensimon, L., et al. (2014) Type 2 Diabetes and the Risk of Mortality among Patients with Prostate Cancer. Cancer Causes Control, 25, 329-338. https://doi.org/10.1007/s10552-013-0334-6

[5] Lee, J., Giovannucci, E. and Jeon, J.Y. (2016) Diabetes and Mortality in Patients with Prostate Cancer: A Meta-Analysis. Springerplus, 5, 1548. https://doi.org/10.1186/s40064-016-3233-y

[6] Karlin, N.J., et al. (2017) Co-Existing Prostate Cancer and Diabetes Mellitus: Implications for Patient Outcomes and Care. Endocrine Practice, 23, 816-821. https://doi.org/10.4158/EP161702.OR

[7] Khan, S., et al. (2016) The Association of Diabetes and Obesity with Prostate Cancer Aggressiveness among Black Americans and White Americans in a Population-Based Study. Cancer Causes Control, 27, 1475-1485.

https://doi.org/10.1007/s10552-016-0828-0

[8] Haggstrom, C., et al. (2017) Prospective Study of Type 2 Diabetes Mellitus, Anti-Diabetic Drugs and Risk of Prostate Cancer. International Journal of Cancer, 140, 611-617. https://doi.org/10.1002/ijc.30480

[9] Atchison, E.A., et al. (2011) Risk of Cancer in a Large Cohort of U.S. Veterans with Diabetes. International Journal of Cancer, 128, 635-643. https://doi.org/10.1002/ijc.25362

[10] Ohwaki, K., et al. (2017) Comorbidities as Predictors of Incidental Prostate Cancer after Holmium Laser Enucleation of the Prostate: Diabetes and High-Risk Cancer. Aging Male, 1-4. https://doi.org/10.1080/13685538.2017.1301417 